\title{
Formation of 7-nm-wide Line\&Spaces in Half Pitch by 3 Dimensional Self-assembly of Nano-dots Using Sphere Type PS-PDMS
}

Sumio Hosaka, Takashi Akahane, Kazumasa Ohyama, Hui Zhang, You Yin, and Hayato Sone

\begin{abstract}
We have formed nanometer-wide lines \& spaces by graphoepitaxy of sphere type polystyrene-poly dimethyl siloxane (PS-PDMS), with a molecular weight (MW) of $14.6 \mathrm{~kg} / \mathrm{mol}$., along electron-beam (EB)-drawn resist guide lines. We have 3-dimensionally ordered the sphere type PS-PDMS by controlling a thickness of the PS-PDMS along improved guide lines to form the line and space pattern. We obtained the thickness dependence on the pattern change such as nano-dot arrays and nano-line $\&$ space patterns. When the thickness increased to about $+4 \mathrm{~nm}$ from the upper thickness for formation of the dot arrays, the line $\&$ space patterns have been formed with about $7 \mathrm{~nm}$ in line width and $14 \mathrm{~nm}$ in pitch.
\end{abstract}

Keywords - Nano-lines, Ordering, Self-assembly, graphoepitaxy, Block copolymer, PS-PDMS.

\section{INTRODUCTION}

Nanoscale devices and ultrahigh-density storage media require the fabrication technology for ultrahighpacked dot arrays [1]-[6] and line \& space patterns [7]-[9]. In order to develop such patterns, there are some methods such as optical stepper, EB (electron beam) drawing [10]-[12], FIB (focused ion beam) machining [13], block copolymer (BCP) self-assembly [1]-[9] and so on. We have studied BCP self-assembly for advanced fabrication in ultra-highly packed magnetic storages and semiconductor devices. In the future, the magnetic storage needs to form high density of patterned media with a size of less than $10 \mathrm{~nm}$. Furthermore, in semiconductor devices, the ITRS lithography-roadmap requires to develop the fabrication method with half-pitches of $12 \mathrm{~nm}$ and $6 \mathrm{~nm}$ in DRAM (dynamic random access memory) and MPU (microprocessing unit) metal minimum lithographically defined half-pitches, respectively, at 2024 [14].

So far, we have developed 5-nm-sized and 10-nm-pitched dot arrays ordering along the fine EB-drawn resist guide lines [15]-[17]. We have also studied on formation of nano-lines along the guide lines by selfassembly using the PS-PDMS (polystyrene-poly dimethyl siloxane) with a molecular weight (MW) of 14.6 $\mathrm{kg} / \mathrm{mol}$. Based on micro-phase separation, these patterns are formed by using BCPs with sphere and cylinder structures. The 2 type structures are determined by selecting molecular weight(MW) of PS-PDMS and the parameters of a product of Flory-Huggins parameter and polymerization and a fraction of PDMS for PSPDMS following the micro-phase separation diagram [18]. Regarding nano-lines formation with 3Dordering in PS-PDMS self-assembly, as a typical work, C. Ross et. al. have shown nanolithography application on trench substrates using cylinder type BCP [19]. On the other hand, the formation using sphere type BCP has not be succeeded yet. We have tried to form line and space patterns with sphere-type PSPDMS (BCP). In this research, we aimed to study 3D-ordering of the PDMS spheres using PS-PDMS selfassembly in graphoepitaxy to form nano-line \& space patterns along the guide lines by controlling the thickness of sphere type BCP of PS-PDMS with MWs of $11.7 \mathrm{~kg} / \mathrm{mol}$. and $2.9 \mathrm{~kg} / \mathrm{mol}$. in PS and PDMS, respectively. 


\section{NANO Line Formation USING 3D (DIMENSION) ORDERING OF NANO DOTS}

In order to fabricate nanometer-wide lines, we adopt an idea to order and stack the BCP dots as arranged the $2^{\text {nd }}$ layer BCP dots line on the $1^{\text {st }}$ layer BCP dots line. Here, we notice (1) the strong adsorption of the PS-terminated guide line for the PS-PDMS dots and (2) high aspect guide line with steep side wall.

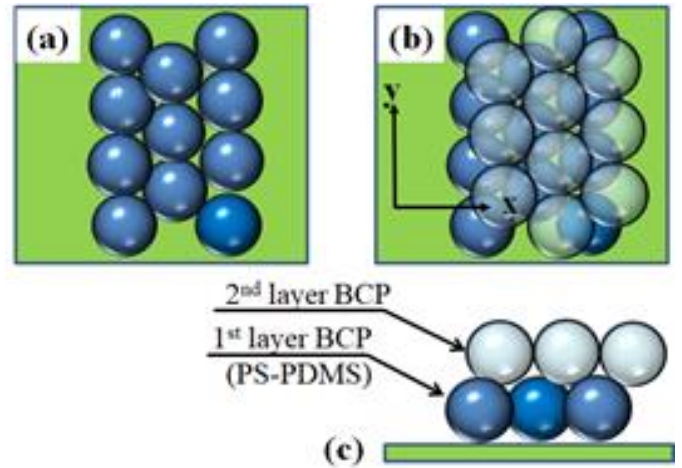

Fig. 1. Scheme for formation of PS-PDMS sphere (dot) arrays on flat substrate without guide line, (a) dot arrays formation in a case of coating thin PS-PDMS film (1 layer dot arrangement), (b) dot arrays formation in a case of thick coating PS-PDMS (2 layers dot arrangement), (c) cross-section of (b).

Fig. 1(a) shows to order PS-PDMS spheres (dots) as line on the flat substrate by controlling thickness of the PS-PDMS film as monolayer. The dot diameter of about $16 \mathrm{~nm}$ is estimated from our experiments [5], [6], [15]. Using thicker film as same as double layers of PS-PDMS sphere, stacking structure of the PSPDMS spheres is formed on the $1^{\text {st }}$ dots pattern. The upper sphere ideally locates on the center of 3 spheres formed as equilateral triangle (Fig. 1(b)). The stacked pattern is not ordered as lines because the $2^{\text {nd }}$ layer spheres are not ordered just on the $1^{\text {st }}$ layer sphere line. On the other hand, using the steep guide line, we make nano-line \& space pattern formed by 3D-ordering the PS-PDMS spheres along steep wall of the guide lines with the sphere type PS-PDMS (Fig. 2). The steep wall means that it is a wall with right or near a right angle to the base surface. The close-section of the guide line is approximately rectangle. Using the guide lines, when coating the PS-PDMS solution as monolayer, the spheres are ordered along the guide lines as dots line (Fig. 2(a)). Moreover, when we coat the thicker PS-PDMS film as double layer, the spheres grow and order as touched with the guide line in X-direction and on low position (saddle) between the ordered $1^{\text {st }}$ layer PS-PDMS spheres along the line in Y-direction (Fig. 2(b)). This arrangement can be considered by characteristics that PS-brushing treated guide line strongly attracts the PS-PDMS spheres in X-direction. Figure 3 shows formation of PDMS sphere arrays and the spheres-piled line \& space pattern by 2-step reactive ion etching (RIE) with $\mathrm{CF}_{4}$-gas for etching PDMS and $\mathrm{O}_{2}$-gas for etching PS for developing of the PDMS spheres latent image. Thus, by controlling the thickness and using the guide lines, we obtain 2 patterns of the PDMS dot arrays pattern (Fig. 3(d)) and the piled PDMS dot line \& space pattern (Fig. 3(e)).

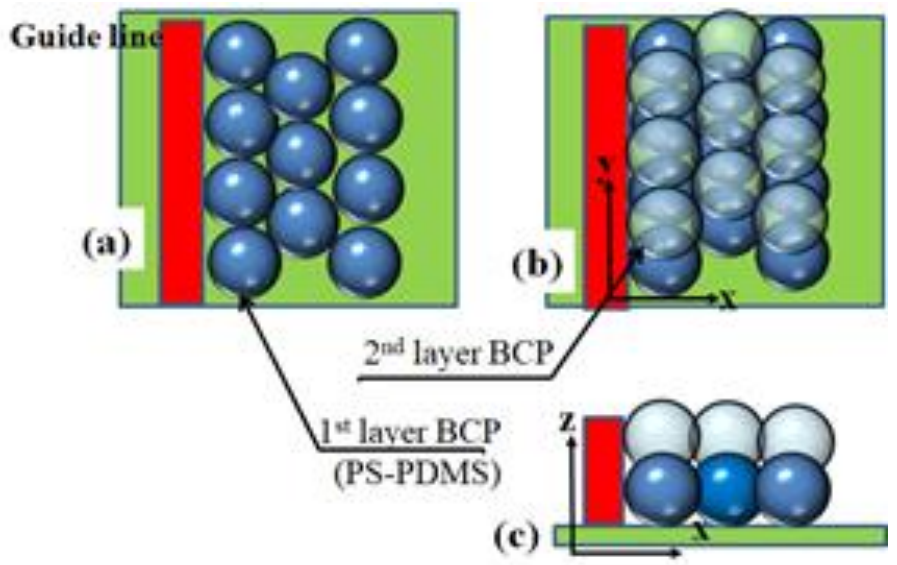

Fig. 2 Scheme for formation of PS-PDMS sphere (dot) arrays and line \& space pattern using guide line, (a) dot arrays formation in a case of coating thin PS-PDMS film (1 layer dot arrangement), (b) line \& space pattern formation in a case of thick coating PSPDMS (2 layers dot arrangement), (c) cross-section of (b).

However, in practice, it is difficult to make the ideal guide lines with the cross-section of rectangle. We have to consider the self-assembly using the guide line with the cross-section of trapezoid (Fig. 4). As shown in Fig. 4(a) and 4(b), the $2^{\text {nd }}$ layer PS-PDMS sphere line is ordered with a shift of half pitch of the $1^{\text {st }}$ layer line pitch in X-direction. As the result, we get the PS-PDMS sphere pattern with no lines (Fig. 
4(c)) as same as that in Fig. 1(b). As described above, we have to use the ideal guide lines with a crosssection of rectangle to form the pattern with PS-PDMS sphere lines and spaces. In order to achieve the arrangement, we have to use high resolution resist patterning of EB-drawing with hydrogen silsesquioxane (HSQ) resist and salty developing [17]. When thickness of PS-PDMS solution is coated on the PS-blushing treated substrate and the guide lines to be equivalent to the double layer, 3D-ordering of PS-PDMS spheres along the guide lines occurs based on strong attraction of the spheres to the guide line wall as described in our work [15] and the others [20], [21] (Figs. 3(e)).

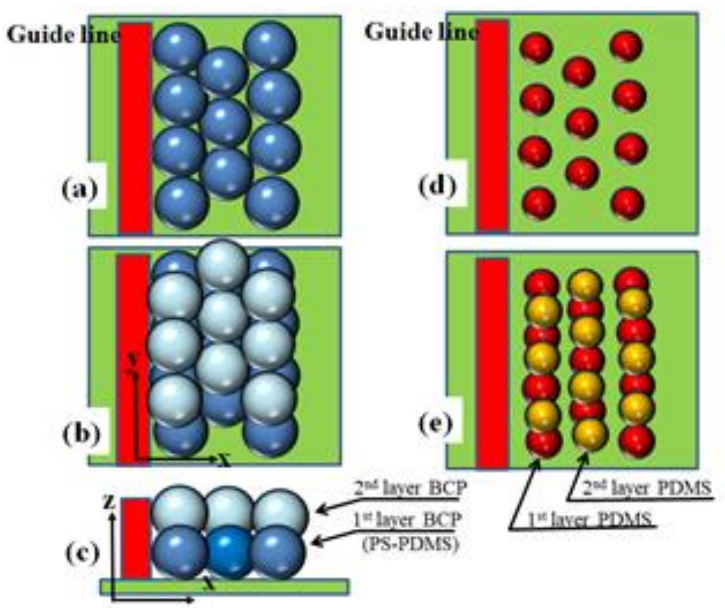

Fig. 3. Scheme for formation of PS-PDMS sphere (dot) arrays and line \& space pattern using guide line, (a) dot arrays formation in a case of coating thin PS-PDMS film (1 layer dot arrangement), (b) line \& space pattern formation in a case of coating thick PSPDMS film (2 layers dot arrangement), (c) cross-section of (b), (d) PDMS dot arrays after RI-etching of (a), and (e) PDMS dots arrays (line structure) after RI-etching of (b).
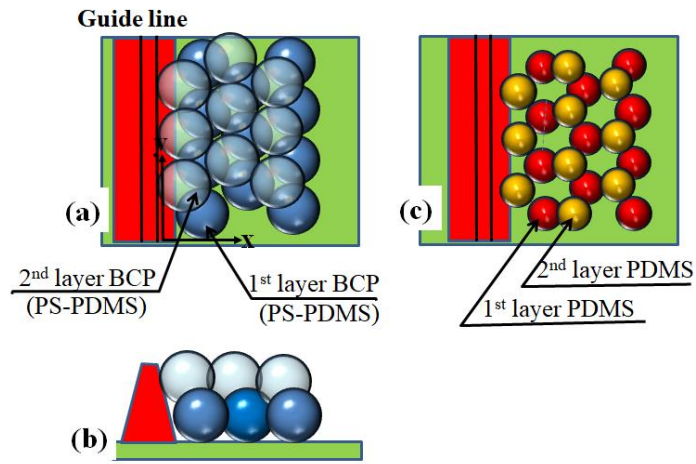

Fig. 4 Schematic model for formation of dot stacked arrays pattern in a case of inclined side wall of guide line as a cross-section of trapezoid, (a) top view of the guide line and stacked PS-PDMS dots, (b) side view of (a), and (c) PDMS dot pattern after RIE of (a).

\section{EXPERIMENT}

In order to form nano-line \& space patterns along the guide lines as described above, we used the PSPDMS with a MW of $11.7 \mathrm{~kg} / \mathrm{mol}$. and $2.9 \mathrm{~kg} / \mathrm{mol}$. in PS and PDMS, respectively. The PS-PDMS has characteristics that the product of Flory-Huggins parameter and polymerization is about 20, and the fraction of PDMS for PS-PDMS is about 0.2. The characteristic means that the structure is selected to self-assembly for the sphere structure sphere, which is located in middle of self-assembled region for the sphere structure in the micro-separation phase diagram. The sphere consists of PS and PDMS molecules, which are covalent bonded. As the PDMS is a core and PS is a cloud layer, we can make the PDMS sphere (dot) arrays by the RIE process. Then, we performed to order the PDMS dots (dot line arrays) or the line arrays (line \& space) along the guide lines.

We performed the experiments as followings, (1) designing and forming of the resist guide lines on flat silicon substrate using EB-drawing with HSQ resist with a thickness of around $25 \mathrm{~nm}$, (2) after forming HSQ resist guide lines, brushing of PS monolayer on the substrate with the resist guide lines, (3) coating of the PS-PDMS solution on the substrate, (4) annealing of the substrate at about $170{ }^{\circ} \mathrm{C}$ for 11 hours, and (5) 2-step reactive ion-etchings of the substrate with $\mathrm{CF}_{4}$ and $\mathrm{O}_{2}$ gases for development of PDMS dot patterns from the latent image. Then, we evaluated the self-assembled patterns on structures and quality of ordering with high resolution SEM (model JSM-6500F made by JEOL). In the experiment, the PS-PDMS solution coatings were done by controlling a spin revolution of 5000 to $8000 \mathrm{rpm}$, which corresponded to $14 \mathrm{~nm}$ to 
$26 \mathrm{~nm}$ in the thickness. The guide line pattern was used with 2 parallel lines. The EB drawing was done with $30 \mathrm{keV}$ high resolution SEM system [11], [12].

\section{EXPERIMENTAL RESULTS AND DISCUSSION}

In the experiments, we obtained some mixed patterns with both nano-dot arrays and nano-lines arrays along the guide lines. Not only the PS-PDMS solution thickness but also the guide line height provided the mixing patterns. The guide line height influences on the PS-PDMS thickness in the guide lines gap. It was clear that the PS-PDMS dot arrays formation began to be created in a region of more than $15 \mathrm{~nm}$ in the HSQ resist thickness for the guide lines [16]. Here, we set constant thickness of the coated HQS resist to about $25 \mathrm{~nm}$ in the EB drawing. Furthermore, we used high resolution EB drawing with salty developer for HSQ resist to get steep side wall of the guide line [12], [16].

Using the condition, we studied the pattern change (dot or line arrays) when changing PS-PDMS solution thickness in coating on the substrate. We obtained SEM images as shown in Fig. 5. The self-assembled pattern changed from dot arrays (Fig. 5(a)) at a thickness of $18 \mathrm{~nm}$ in the PS-PDMS solution to line $\&$ space pattern (Fig. 5(c)) at $22 \mathrm{~nm}$ through mixing pattern (Fig. 5(b)) at $20 \mathrm{~nm}$ when the thickness increased approximately from $14 \mathrm{~nm}$ to $25 \mathrm{~nm}$. In addition, we obtained the pattern change property as shown in Fig. 6. The figure also shows these patterns can be determined by PS-PDMS thickness. The horizontal axis indicates thickness deviation from the thickness at a center of the region to obtain the dot and line mixing pattern, and the vertical axis indicates the gap of the guide lines. The dot arrays appeared when the thickness decreased from that at center of the mixing region while the line \& space pattern appeared when the thickness region increased from that. The width of mixing region is about $2 \mathrm{~nm}$. These results clarified that the thickness of the coated PS-PDMS solution controlled nano-patterns, either dot arrays or line \& space pattern in the guide lines gap. Furthermore, the property is given even in wide gap between the guide lines of about $600 \mathrm{~nm}$.
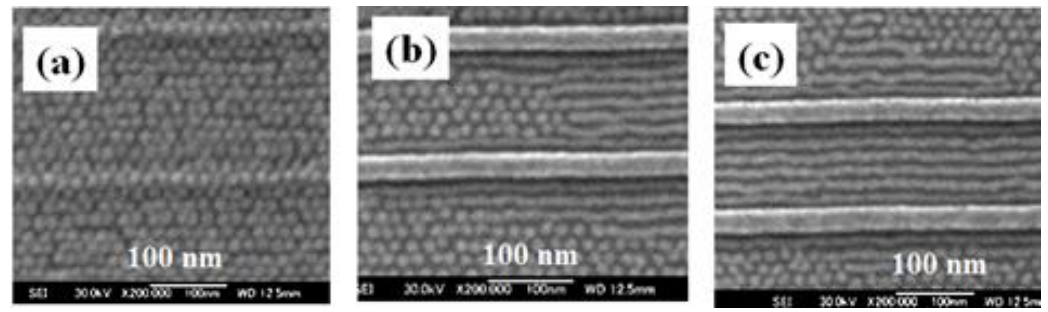

Fig. 5. SEM images of PDMS sphere (dot) arrays and line \& space pattern formed with guide line after RIE, (a) PS-PDMS dot pattern in a case of coating thin PS-PDMS film (about $18 \mathrm{~nm}$ ), (b) PDMS dot and line mixing pattern in a case of coating PS-PDMS film with a little thicker than that in (a) (about $20 \mathrm{~nm}$ ), and (c) PDMS line \& space pattern between guide lines with thick PS-PDMS film (about $22 \mathrm{~nm}$ ).

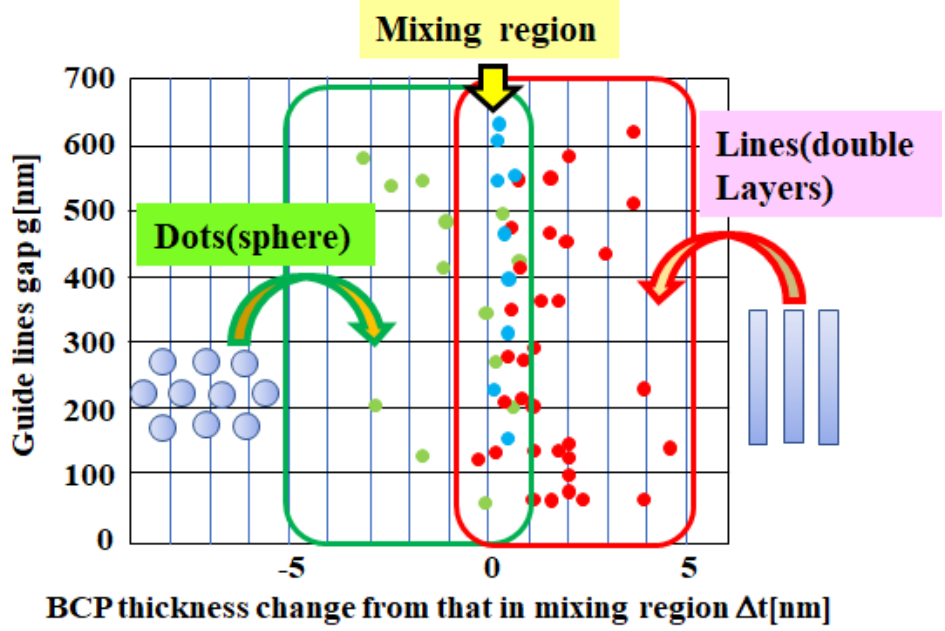

Fig. 6. Relationship between BCP (PS-PDMS) coated film thickness and the pattern change from dot arrays to line \& space patterns (red marks: line\&space(L\&S) pattern, green marks: dots lines and blue marks: mixing pattern with L\&S pattern and dots lines).

Fig. 7 shows SEM images of self-assembled line \& space patterns along the guide lines with a gap of 84 $\mathrm{nm}$ to $135 \mathrm{~nm}$. The line width is also about $7 \mathrm{~nm}$ (half-pitch) with a pitch of about $14 \mathrm{~nm}$. On the PDMS lines in the figure, rugged structure like dots is watched. The structure means that the lines consisted of dots as shown in Fig. 3(e). As shown in Fig. 5(b), the mixing region may be caused by change of the PS-PDMS thickness in the gap. One of the changes can be considered to be due to capillary force between the base 
surface and the side walls of the guide lines. In further experiments, we have succeeded to form maximum 37 nano-lines in the gaps of about $587 \mathrm{~nm}$ as shown in Fig. 8.
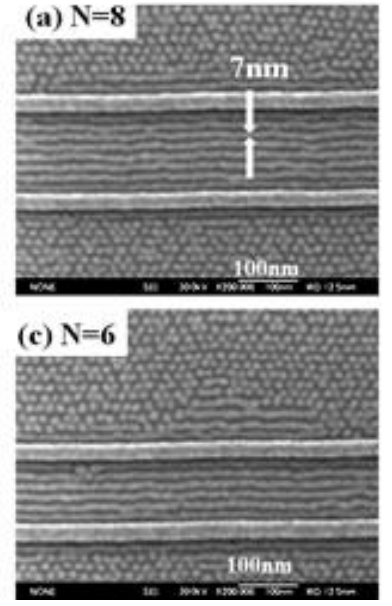
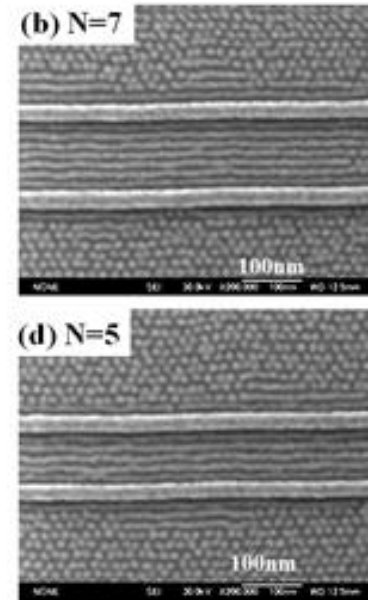

Fig. 7. SEM images of line \& space patterns formed in the guide line gap by self-assembling using PS-PDMS with a MW of 14.6 $\mathrm{kg} / \mathrm{mol}$.; (a) 8 lines in a gap of $135.5 \mathrm{~nm}$, (b) 7 lines in $112.8 \mathrm{~nm}$, (c) 6 lines in $103.4 \mathrm{~nm}$, and (d) 5 lines in $84.8 \mathrm{~nm}$.

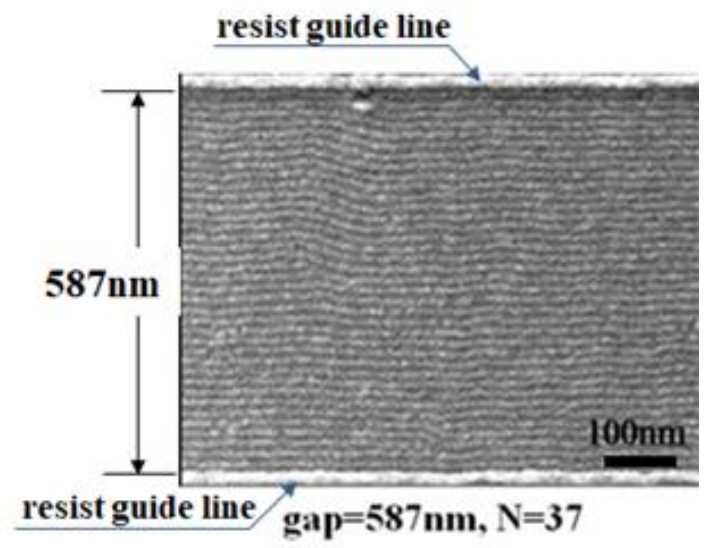

Fig. 8. SEM image of line \& space patterns with 37 lines formed in the guide lines with wide gap of $587 \mathrm{~nm}$ by self-assembly using PS-PDMS with a MW of $14.6 \mathrm{~kg} / \mathrm{mol}$.

From the figure, we confirmed that the PS-terminated (brushing) guide lines have strongly attractive force to the PS-PDMS spheres. Furthermore, the fact that the line \& space pattern was formed with the PSPDMS spheres in the gap of guide lines by 3D-ordering indicates that the guide line was made as same as rectangle in a cross-section (Fig. 3(b) and 3(c)). We can consider that the cross-section is formed by salty developing in EB drawing with HSQ resist. In our previous study on the EB drawing, the EB drawing method has improved the contrast of HSQ-resist EB-drawing and high gamma-value developing [12]. This experimental result agrees with the consideration as described in Fig. 3 and 4.

The method can be expected to contribute to nano-lithography for half-pitch of $7 \mathrm{~nm}$. When we use light PS-PDMS, for example, about $8.5 \mathrm{~kg} / \mathrm{mol}$. [16], we can consider to get half-pitch of $5 \mathrm{~nm}$, which will be required in ITRS in 2029 [14].

\section{CONCLISION}

We have studied on formation of nano-lines ordering along guide lines using graphoepitaxy with sphere type block copolymer (BCP) of polystyrene-poly dimethyl siloxane (PS-PDMS) self-assembly with a MW of $14.6 \mathrm{~kg} / \mathrm{mol}$ and EB drawn HSQ-resist guide lines. Especially, we studied to form nano-dot and nanolines along the guide lines by controlling of the thickness of the BCP coated on substrate. We obtained the thickness dependence on the pattern of nano-dots or nano-lines, which was ordered along the guide lines. Furthermore, when the suitable thickness was controlled for formation to get nano-line by stacked $2^{\text {nd }}$ nanodot line just on $1^{\text {st }}$ nano-dot line, we obtained to form nano-line arrays (line $\&$ space pattern) with a width of about $7 \mathrm{~nm}$ and a pitch of $14 \mathrm{~nm}$. In addition, we have demonstrated to form 5 to 8 nano-lines in the guide line gaps of about 84 to $135 \mathrm{~nm}$. Furthermore, we have shown the possibility to make 37 nano-lines with the guide lines gap of $587 \mathrm{~nm}$. 


\section{ACKNOWLEDGMENT}

We would like to thank Dr. M. Huda and Mr. T. Komori in Gunma University for great technical contributions in fabrication of the self-assembled dot arrays and EB drawing.

\section{FUNDING}

This work was financially supported by Grant-in-Aid for Young Scientists and Grant-in-Aid for Scientific Research from the Ministry of Education, Culture, Sports, Science and Technology of Japan (No. 24686042, No. 21710135, No. 24360003, No. 26282143, No. 18H03547, No. 19K23598), JST Sentan Grant Number H24sentan181-25, and Grants-in-Aid for JSPS Research Fellow No. JP17F17058.

\section{CONFLICT OF INTEREST}

Authors declare that they do not have any conflict of interest.

\section{REFERENCES}

[1] I. Bita, J. Yang, Y. Jung, C. Ross, C. Thomas, K. Berggren. Graphoepitaxy of Self-Assembled Block Copolymers on TwoDimensional Periodic Patterned Templates, Science, 2008;321: 939-943.

[2] M. Sakurai, Y. Kamata, H. Hieda. Patterned Media Nanosize Magnetic Dot Arrays Patterned along Tracks. Toshiba Rev. 2002;57: 52-55.

[3] H. Kitano, S. Akasaka, T. Inoue, F. Chen, M. Takenaka, H. Hasegawa, H. Yoshida, H. Nagano. Control of the Microdomain Orientation in Block Copolymer Thin Films with Homopolymers for Lithographic Application. Langmuir, 2007;23: 6404-6410.

[4] N. Kihara, R. Yamamoto, N. Sasao, T. Shimada, A. Yuzawa, T. Okino, Y. Ootera, Y. Kamata, A. Kikitsu. Fabrication of 5 Tdot/in. ${ }^{2}$ bit patterned media with servo pattern using directed self-assembly. J. Vac. Sci. Technol. B30, 06FH02 (1-5), 2012.

[5] S. Hosaka, T. Akahane, M. Huda, T. Tamura, Y. Yin, N. Kihara, Y. Kamata, A. Kikitsu. Long-range-ordering of self-assembled block copolymer nanodots using EB-drawn guide line and post mixing template. Microelectron. Eng, 88, 2571-2575 (2011).

[6] Sumio Hosaka, Takashi Akahane, Miftakhul Huda, Hui Zhang, and You Yin. Ordering of 6-nm-sized nanodot arrays with 10nm-pitch using self-assembled block copolymers along electron beam-drawn guide-lines. Microelectronics Engineering. 2014;123:54-57.

[7] E. Edwards, M. Muiier, M. Stoykovich, H. Solak, J. de Pablo, P. Nealey. Dimensions and shapes of block copolymer domains assembled on lithographically patterned substrates. Macromolecules, 2007;40:90-96.

[8] S. Kim, H. Solak, M. Stoykovich, N. Ferrier, J. de Pablo, P. Nealey. Epitaxial self-assembly of block copolymers on lithographically defined nanopatterned substrates. Nature, 2003;424: 411-414.

[9] E. Edwards, M. Stoykovich, H. Solak, P. Nealey. Long-range order and orientation of cylinder-forming block copolymers on chemically nanopatterned striped surfaces. Macromolecules 39, 3598-3607 (2006).

[10] Z. Mohamad, M. Shirai, H. Sone, S. Hosaka, M. Kodera. Formation of dot arrays with a pitch of $20 \mathrm{~nm} \times 20 \mathrm{~nm}$ for patterned media using $30 \mathrm{keV}$ EB drawing on thin calixarene resist. Nanotechnology, 2008;19, 025301(1-4).

[11] S. Hosaka, Y. Tanaka, M. Shirai, Z. Mohamad, Y. Yin. Possibility of forming 18-nm-pitch ultrahigh density fine dot arrays for 2 Tbit/in. ${ }^{2}$ patterned media using 30-keV electron beam lithography. Jpn. J. Appl. Phys., 2010;49, 046503 (1-3).

[12] T. Komori, H. Zhang, T. Akahane, Z. Mohamad, Y. Yin, S. Hosaka. Electron Beam Lithography of $15 \times 15 \mathrm{~nm}^{2}$ Pitched Nanodot Arrays with a Size of Less than $10 \mathrm{~nm}$ Using High Development Contrast Salty Developer. Jpn. J. Appl. Phys., 2012;51, 06FB02 $(1-4)$.

[13] C. T. Rettner, M. E. Best, B. D. Terris. Patterning of granular magnetic media with a focused ion beam to produce single-domain island at >140Gbit/in², IEEE Tr. Mag. 2001;37, 1649-1651.

[14] https://www.semiconductors.org/wp-content/uploads/2018/06/1_2015-itrs-2.0_lithography-.pdf.

[15] S. Hosaka T. Akahane, M. Huda, H. Zhang, Y. Yin. Controlling of 6-nm-sized and 10-nm-pitched dot arrays ordered along narrow guide lines using PS-PDMS self-assembly. ACS Applied Materials Interface, 2014;6:6208-6211.

[16] H. Zhang, S. Hosaka, and Y. Yin. Ordering of Self-assembled 5-nm-Diameter Poly(dimethylsiloxane) Nanodots with Sub-10 nm Pitch using Ultra-Narrow Electron-Beam-Drawn Guide Lines and Three-Dimensional Control. Appl. Phys. Lett, 2014;104:093107.

[17] T. Komori, M. Huda, T. Akahane, M. Masuda, J. Liu, Z. Mohamad, Y. Yin, and S. Hosaka. Ordering of self-assembled nanodots improved by guide pattern with low line edge roughness for 5 Tbit/in.2 patterned media. Key Engineering Materials, 2014;596:78-82.

[18] A. N. Semenov. Contribution to the theory of microphase layering in block-copolymer melts. Sov. Phys. JETP, 1985;61:733742 .

[19] Y. S. Jung and C. A. Ross. Orientation-controlled self-assembled nanolithography using a polystyrene-polydimethylsiloxoane block copolymer. Nano Lett. 2007;7:2046-2050.

[20] V. P. Chuang, J. Y. Cheng, T. A. Savas, and C. A. Ross. Three-dimensional self-assembly of spherical block copolymer domains into V-shaped grooves. Nano Lett., 2006;6:2332-2337.

[21] C. A. Ross, K. K. Berggren, J. Y. Cheng, Y. S. Jung, and J. Chang. Three-dimensional nanofabrication by block copolymer selfassembly. Adv. Mater., 2014;26:4386-4396. 


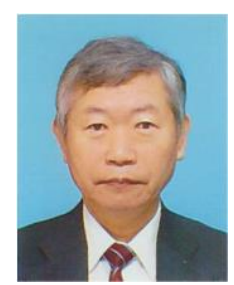

Sumio Hosaka Sumio hosaka was born in Japan in 1948. He received the Ph. D. degree in Engineering from Waseda University, Tokyo, in 1983.

He joined the Hitachi Central Research Lab. (HCRL), Co. Ltd. Kokubunji, Tokyo in 1971, working in semiconductor lithography using electron, ion and light beams, nano-metrology using electron beam microscopy and scanning probe microscopy (SPM) techniques, in HCRL and terabyte huge storage using SPM in the Hitachi Advanced Research Lab. (HARL). After he joined the Hitachi Kenki Co., Tsuchiura, Ibaraki in 2000, working in-line atomic force microscope products based on his invention of first optical lever type atomic force microscope at 1987, he joined to department of electronic engineering, Gunma University, Kiryu, Gunma, in 2001. Then, he worked in division of electronics and informatics, faculty of science and technology in Gunma University. Since 2001, He has been professor in the university, and was working on nano devices such as quantum conductance atomic switch and phase change memory, nano lithography and fabrication such as EB drawing, self-assembled nano dot arrays and patterned media in huge magnetic storage, and nano metrology based on atomic force controlling, near-field light, Raman spectroscopy and polarized light image in SPM applications. Then, he retired Gunma University in 2014, and he is proffessor emeritus in faculty of science and technology Gunma University. 